\title{
The challenges associated with short volume sodium citrate blood samples
}

\author{
Mr Danny Gaskin, BSc (Hons), CertHE, LIBMS, AMRSB, Biomedical Scientist, Haematology \\ and Transfusion Science.
Mr Ola Yahaya, MSc, MIBMS AMLSCN. Specialist Biomedical Scientist, Haematology and Transfusion Science.

In the laboratory, we often receive phone calls from our clinical colleagues with questions about decisions we have made that might affect their patient's care. For example, enquiries about why we have rejected a sample or why we were unable to provide a particular result due to a pre-analytical or technical error.

In this series of short articles, we want to try and address some of the most common questions we get asked, with the aim of educating the wider workforce in a way that is simple to understand, about the decisions we make in the laboratory.

We bope that with a greater understanding, the number of avoidable errors might be reduced.

In this article, we explore the pre-analytical challenges associated with short volume sodium citrate anti-coagulated blood samples and why these must be rejected.

In the summer of 2018 , we curated the @NHS Twitter account for a week, raising the profile of healthcare scientists up and down the country, reaching an online audience of around 1.9 million. We used the platform to engage with patients, colleagues and the general public about the role of healthcare scientists and present a transparent insight into what happens in a hospital pathology laboratory.

Surprising to us, the most popular tweet we put out was a question about a blood sample we'd rejected earlier that afternoon. We asked, "would you be disappointed if this blood sample was rejected?". We included a photograph of an anonymised under filled (approximately $65 \%$ filled) sodium citrate sample tube, used to collect blood for coagulation studies. The response we got from clinicians was huge - apparently this is quite the topic for debate, however for laboratory staff, the reason for rejection is clear, and in this article, we'd like to share with you why that is.

Whilst the majority of those that engaged seemed to agree that the sample was under filled and, therefore would be rejected - not everybody understood why. Many went on to share stories of frustration around 'difficult to bleed' patients and persistently rejected blood samples, and quite rightly asked the question, "why is it so important for these blood samples to be filled to the line?

Firstly, let's go back to basics with blood tubes. These tubes have different coloured lids for good reasons. It's important to know that the different colours represent different additives inside the tubes and these colours are denoted by an international standard (ISO 6710:2017). ${ }^{1}$ These are designed to preserve and protect the integrity of blood samples pre-, post- and during analysis. It is important that the appropriate additive is selected when collecting the blood sample for a particular pathology investigation. $^{2}$ Selecting the wrong colour top, and therefore the wrong additive could result in clinically significant inaccuracies in the results that are reported, with potentially serious medical consequences.

So, let's take a look at the sodium citrate tubes. These tubes contain a measured amount of buffered trisodium citrate solution. ${ }^{3,4}$ In the healthcare setting you'll often hear it referred to as 'sodium citrate' or just 'citrate'.

The natural process of blood clotting requires calcium ions $\left(\mathrm{Ca}^{++}\right){ }^{5}$ The sodium citrate in this tube, when mixed with freshly collected blood, prevents this process by withholding the $\mathrm{Ca}^{++}$in the blood sample. ${ }^{6,7}$ When we receive the blood sample in the laboratory, part of our procedures for investigating a clotting profile is to reintroduce $\mathrm{Ca}^{++}$from a synthetic source which is usually a standard reagent.

Common coagulation tests such as prothrombin time (PT) and activated partial thromboplastin time (APTT) are performed by adding a measured amount of calcium to the plasma of the anti-coagulated blood sample, this is expected to replenish the withheld $\mathrm{Ca}^{++}$, so that the laboratory-induced clotting can be initiated and measured. As this is a measure-for-measure test, it is critical to mix the right amount of blood with the right amount of citrate. ${ }^{8}$ Tube manufacturers provide a fixed and validated volume of sodium citrate which is to be mixed with the patient's blood, at a fixed ratio of 1:9 (citrate solution to whole blood). The marker line you see on the tubes is a guide to the volume of blood required to achieve this ratio. In BD tubes (the ones we use at Morecambe Bay), this is commonly a frosted line in the plastic, which is the minimum fill line. In other tubes, and tubes from other manufacturers, this may be the nominal (i.e. $100 \%$ fill) line.

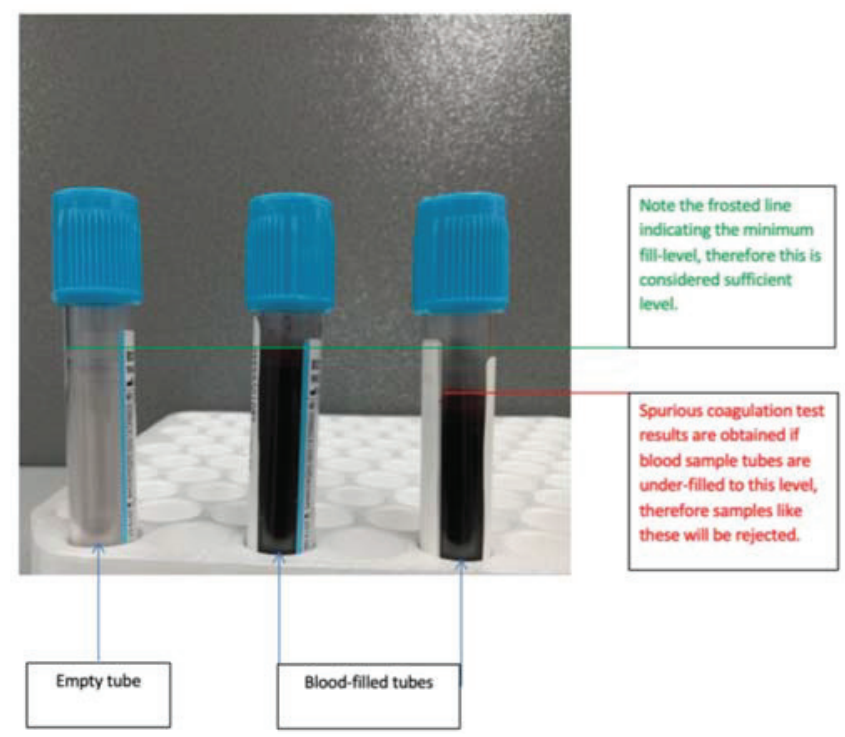

The healthcare staff working in the clinical setting who are tasked with collecting blood samples can unknowingly alter this ratio by collecting a volume of blood that is less than what is required. When this happens, the sodium citrate to blood ratio is altered and this can become an issue. 
This means that there will be left-over citrate, which is still active and has the potential to interfere with the $\mathrm{Ca}^{++}$that will be added upon testing. This may create uncertainty in the validity of the results we produce in the laboratory. ${ }^{8}$ For instance, the possibility of an extended PT, as the $\mathrm{Ca}^{++}$ we add will take time to reverse the citrate effect leading to falsely-prolonged clotting times. Similarly, collecting blood from a wing set or IV extension can draw air into the tube, resulting in inadvertent under-filling and, therefore, an insufficient volume of blood being collected.

It's worth noting that, overfilling these tubes can also cause undesirable alterations to the ratio of citrate and blood, ${ }^{8}$ however, in tubes with a vacuum, you can only significantly over-fill the tubes by taking the cap off to fill - this is against manufacturer advice ${ }^{9}$ and should never be done.

So, is there any room for leniency? No, not really. Our advice to anybody collecting blood at Morecambe Bay into a sodium citrate tube is to always collect sufficient volume to at least meet or exceed the frosted guide line. Studies have demonstrated that clinically significant changes to results occur at different levels of under-fill for different tests.

For context, let's take the APTT. Clinically significant inaccuracies to the result can occur when less than $89 \%$ of the required volume is collected. ${ }^{10}$ Similarly, you can expect clinically significant inaccuracies to fibrinogen results at around $78 \%$ of the required volume, whereas more specialist tests such as a Factor VIII assay comes in at around $67 \% .{ }^{10}$

It's for reasons like these we occasionally have to (regrettably) reject specimens, because, like you, we (Biomedical Scientists) as professionals are heavily regulated and have standards of proficiencies that we must abide to, and although we rarely see them, we take great pride in providing the best care we can to our patients.

When you are challenged with difficult peripheral venous access, it may be difficult to aspirate a sufficient volume of blood. Many of you shared your stories with us on Twitter about patients that were 'difficult to bleed' for all sorts of reasons, and some of you asked, what if it simply isn't realistically possibly to draw enough blood?

Firstly, for the best clinical outcomes, it is important to follow your specimen collection tube manufacturer's instructions as well as your organisation's venepuncture policies, where applicable.

You may then want to consider selecting a different size of sodium citrate tube. For a 'standard' sodium citrate tube, $2.7 \mathrm{ml}$ of whole blood is required. As discussed earlier, it is this specific volume of blood that, when mixed with the sodium citrate inside the tube, achieves the required ratio of citrate to blood to perform the tests. Now, depending on the manufacturer that the organisation you work for acquires blood tubes from, there may be other sizes available. Here at Morecambe Bay, we're able to provide our clinical colleagues with tubes that require as little as $1.8 \mathrm{ml}$ of blood whilst still achieving the required ratio, and conversely, for investigations that might require a greater plasma yield, we're also able to provide a larger tube that can collect $4.5 \mathrm{ml}$ of blood. You should contact the Pathology department at your hospital to enquire about availability.

To summarise, unsatisfactory results provide little value in the diagnosis or monitoring of a patient and can in some cases cause harm, when no action or unnecessary action is taken on the back of an inaccurate result. No specimen is ever rejected lightly, and when specimens are rejected, it's always in the best interests of the patient behind the sample.

\section{References}

1. International Organization for Standardization. Single-use containers for human venous blood specimen collection. ISO, 2017. Available at: https:// www.iso.org/obp/ui/\#iso:std:69659:en. (accessed 2.3.19).

2. Lippi G, Salvagno GL, Montagnana M, LimaOliveira G, Guidi GC, Favaloro EJ. Quality standards for sample collection in coagulation testing. Seminars in Thrombosis and Hemostasis 2012;38(6):565-575.

3. Favaloro EJ, Lippi G, Adcock DM. Preanalytical and postanalytical variables: the leading causes of diagnostic error in hemostasis? Seminars in Thrombosis and Hemostasis 2008;34(7):612-634.

4. $\mathrm{Pa}$ W. Clinical and Laboratory Standard Institute C. Methods for dilution antimicrobial susceptibility tests for bacteria that grow aerobically. Approved standard M7-A7. Clinical and Laboratory Standard Institute. 2006.

5. Mikaelsson M.E. (1991) The role of calcium in coagulation and anticoagulation. In: Sibinga C.T.S., Das P.C., Mannucci P.M. (eds) Coagulation and blood transfusion. developments in hematology and immunology, vol 26. Boston, MA: Springer, 1991.

6. Sabbatani L. Calcium et citrate trisodique dans la coagulation du sang, de la lymphe et du lait. Arch Ital Biol 1901;36:397-415.

7. Pekelharing CA. Ueber die Gerinnung des Blutes. 1. DMW-Deutsche Medizinische Wochenschrift 1892;18(50):1133-6.

8. Peterson P, Gottfried EL. The effects of inaccurate blood sample volume on prothrombin time (PT) and activated partial thromboplastin time (aPTT). Thrombosis and Haemostasis 1982;47(02):101-3.

9. BD. General BD Vacutainer ${ }^{\circledR}$ blood collection tubes FAQ. Available at: https://www.bd.com/ en-us/offerings/capabilities/specimen-collection/ blood-specimen-collection/venous-collection/bdvacutainer-blood-collection-tubes/vacutainer-bloodcollection-tube-faq/general-tubes-faq (accessed 3.3.19).

10. Reneke J, Etzell J, Leslie S, Ng V, Gottfried E. Prolonged prothrombin time and activated partial thromboplastin time due to underfilled specimen tubes with $109 \mathrm{mmol} / \mathrm{L}$ (3.2\%) citrate anticoagulant. American Journal of Clinical Pathology. 1998;109(6):754-757.

\section{Acknowledgments}

1. Adam Stretton, Clinical Manager, UK \& Ireland, BD Life Sciences, Pre-analytical System

This article was originally published in The Nursing Standard on 30th January 2019.

Correspondence to: dtgaskin@outlook.com 